\title{
Evaluation of post-Holmium laser enucleation of the prostate hospital readmissions
}

\author{
(1) Eymen Gazel${ }^{1}$, (1) Engin Kaya², (1) Serdar Yalçın², (1) Sercan Yılmaz², (1) Selahattin Bedir², (1) Mehmet Yılmaz², \\ (1) Lütfi Tunç ${ }^{4}$
}

\author{
${ }^{1}$ Acibadem Ankara Hospital, Clinic of Urology, Ankara, Turkey \\ 2University of Health Sciences Turkey, Gülhane Training and Research Hospital, Clinic of Urology, Ankara, Turkey \\ 3Zile State Hospital, Clinic of Urology, Tokat, Turkey \\ ${ }^{4}$ Gazi University Faculty of Medicine, Department of Urology, Ankara, Turkey
}

\section{Date submitted: \\ 18.03.2020}

Date accepted:

16.04.2020

Online publication date:

15.09.2020

\section{Corresponding Author:}

Eymen Gazel, MD, Acibadem Ankara Hospital, Clinic of Urology, Ankara,

Turkey

eymen_gazel@yahoo.com

ORCID:

orcid.org/0000-0003-3774-9982

Keywords: After, post, HoLEP, readmission, complication

\begin{abstract}
Aims: Re-admission to the hospital after operation and discharge has become a topic of increasing interest in recent decades and is being studied due to its impact on countries' medical costs. We tried to examine the rates and causes of re-admission to the hospital in 30 days in patients who performed Holmium Laser Enucleation of the Prostate (HoLEP), a popular minimally invasive surgery in recent years.
\end{abstract}

Methods: Patients who underwent HoLEP between July 2017 and April 2019 were retrospectively reviewed. Reasons and rates of re-admission to the hospital in the first 30 days of 300 patients meeting the inclusion criteria, and the way managing the situations were recorded.

Results: In our study, readmission after HoLEP was observed in 26 patients (26/300) as 8.67\% rate with nonspecific and specific causes. Other than nonspecific reasons, hematuria requiring or not requiring transfusion was the most common cause of readmission (2.3\%). The second most common reason for readmission was acute urinary retention requiring three-day antiinflammatory therapy and recatheterization (1.67\%). Urinary tract infection (1.33\%) requiring oral or intravenous antibiotic therapy was the third most common specific cause.

Conclusions: In the literature, readmission rates after HoLEP change between $5.5 \%$ and $17.8 \%$. Readmission rate in our study was $8.67 \%$, which is compatible with the literature. Knowing the reasons and rates of readmission after HoLEP is important to predict early complications after surgery and to manage these complications.

\section{Introduction}

Lower urinary tract symptoms related to benign prostatic obstruction continues to be important as one of the most prevalent health problems observed among adult men (1). Although it is not included in the guidelines as the gold standard yet, use of laser in the treatment of benign prostate hyperplasia $(\mathrm{BPH})$ is becoming more prevalent as a minimally invasive approach alternative to Transurethral Resection of the Prostate (TUR P) (2,3). It is revealed that Holmium Laser Enucleation of the Prostate (HoLEP), which is a minimally invasive and prostate-independent method, has surgical results comparable to traditional TUR $P$ and has low perioperative and postoperative complications and good surgical results in $\mathrm{BPH}$ surgical treatment $(2,4,5)$.

Readmission to the hospital after operation and discharge has become an increasing interest in the last decades and it is being analyzed due to its effect on medical expenses of countries (6). Moreover, hospital readmission rates are being evaluated as a criterion of the quality of healthcare services in the Western countries $(6,7)$. In the present study, we aimed to present the hospital readmission incidences and causes within 30 days in patients we performed HoLEP, which is a recently popular minimally invasive surgery. We think that our study will contribute to the literature since the number of studies on 
the reasons and rates of re-admission to hospital after HoLEP surgery is very low.

\section{Methods}

Patients who had HoLEP performed between July 2017 and April 2019 in Acibadem Ankara Hospital and Gülhane Training and Research Hospital were retrospectively analyzed. The study protocol was approved by Gazi University Ethical Committee, Turkey (approval number: 2020-150) and complied with the Helsinki Declaration. Diagnosis of obstruction was confirmed with urine flow rate, post-void residual (PVR) urine and preoperative International Prostate Symptom Score (IPSS). Inclusion criteria for HoLEP surgery were IPSS of $>8, \leq 15 \mathrm{~mL}$ / sec maximum urine flow rate, and PVR of $\geq 50 \mathrm{~mL}$. Prior to the HoLEP surgery, all cases had cystoscopy performed in order to examine obstruction, bladder trabeculation, urethral and bladder pathologies and to exclude bladder tumor. A total of 9 patients were excluded from the study. These patients included three prostate cancer patients, two bladder cancer patients, one neurogenic bladder patient, and three urethral stricture patients. All patients signed an informed consent form prior to the surgery. The HoLEP procedure and technique performed were previously explained by the authors (8). Causes and rates of hospital readmissions in 300 patients, who underwent HoLEP and who, afterwards, had catheter removed and were discharged, were recorded along with the methods of managing the encountered situations.

\section{Statistical Analysis}

The Statistical Package for Social Sciences 23.0 software (SPSS 23.0, Chicago, IL, United States of America) was utilized. Descriptive statistics of scale samples and peroperative outcomes were expressed as mean \pm standard deviation or median \pm interquartile range.

\section{Results}

Causes and rates of hospital readmissions in 300 patients, who had HoLEP performed between July 2017 and April 2019, were evaluated along with the management of these situations. Patients' characteristics are presented in Table 1. The mean age was 62.6 years and the mean prostate size was $88.27 \mathrm{~g}$. The difference between preoperative and postoperative PSA values was significant statistically $(p<0.001)$. Causes and rates of readmissions and management methods are presented in Table 2. It was observed that highest rates of postoperative readmission were due to nonspecific symptoms such as postoperative emesis, fever, pain, etc. (2.3\%). Other than these nonspecific symptoms; hematuria requiring or not requiring transfusion and requiring surgical intervention was observed as the most prevalent cause of readmission $(2.3 \%)$, which was followed by re-catheterization and acute urinary retention requiring three-day anti-inflammatory treatment (1.67\%). Urinary tract infection requiring oral or intravenous antibiotic treatment $(1.33 \%)$, clot retention $(0.67 \%)$, and hematuria requiring transfusion $(0.67 \%)$ were observed. Temporary hematuria not causing low hemoglobin $(1.67 \%)$, deep vein thrombosis $(0.33 \%)$ were observed with less prevalence.

\section{Discussion}

Hospital readmission request and acceptance rates are being analyzed in the United States of America due to their effects on increasing medical costs (7). It has been shown that readmission request and acceptances to Centers for Medicare and Medicaid Services in United States of America cost 17.5 billion dollars in 2010 and moreover, $27 \%$ of the readmission requests and acceptances were preventable $(7,9)$.

There are studies in the literature presenting the readmission causes, incidences, and predictive factors after open or endoscopic urologic surgeries $(6,7,10-12)$. In a study, the most prevalent reasons for readmission within 30 days after TUR $P$ were listed as hematuria $(n=11 ; 6.8 \%)$, fever/urinary tract infections (UTI) $(n=7 ; 4.3 \%)$ and acute urinary retention $(n=5$; $3.1 \%$ ) (6). In a study, in which the readmission rates of the urologic surgeries performed as "outpatient" were analyzed, Rambachan et al. (9) observed the readmission rate in patients

Table 1. Preoperative measures and postoperative hemoglobin level, hemoglobin drop and prostate specific antigen levels

\begin{tabular}{|llll}
\hline Value & Mean & Minimum & Maximum \\
\hline Age (years) & 62.6 & 46 & 86 \\
\hline BMI (kg/cm $)$ & 23.89 & 17.10 & 33.20 \\
\hline Prostate size $(\mathbf{g})$ & 88.27 & 21 & 400 \\
\hline PSA-pre $(\mathbf{n g} / \mathbf{m L})$ & 4.4 & 0.33 & 18.4 \\
\hline PSA-post $(\mathbf{n g} / \mathbf{m L})$ & 0.92 & 0.14 & 2.14 \\
\hline Hgb-pre $(\mathbf{g} / \mathbf{d L})$ & 14.29 & 10.14 & 17 \\
\hline Hgb-post $(\mathbf{g} / \mathbf{d L})$ & 13.48 & 9.31 & 16.79 \\
\hline Hgb-drop $(\mathbf{g} / \mathbf{d L})$ & 0.49 & 0.24 & 1.16 \\
\hline Statistically analyzed with Mann-Whitney U test; another analyzed with Wilcoxon test. & & \\
\hline BMl: Body mass index, Hgb: Hemoglobin, PSA: Prostate specific antigen & & & \\
\hline
\end{tabular}




\begin{tabular}{|c|c|c|}
\hline $\begin{array}{l}\text { Reasons for readmission within } 30 \text { days } \\
\text { after discharge }\end{array}$ & n (\%) & Management \\
\hline Acute urinary retention & $5(1.67 \%)$ & 3-day anti-inflammatory oral therapy and recatheterization \\
\hline Urinary system infection & $4(1.33 \%)$ & Oral or Intravenous antibiotic treatment \\
\hline Clot retention & $2(0.67 \%)$ & $\begin{array}{l}\text { Clot drainage, by urethral catheter irrigation or by cystoscopic } \\
\text { intervention }\end{array}$ \\
\hline Hematuria (requiring transfusion) & $2(0.67 \%)$ & Transfusion \\
\hline $\begin{array}{l}\text { Temporary hematuria (no transfusion } \\
\text { required) }\end{array}$ & $5(1.67 \%)$ & Follow-up-observation \\
\hline Deep vein thrombosis & $1(0.33 \%)$ & Anticoagulant therapy, cardiovascular surgery consultation \\
\hline $\begin{array}{l}\text { Postoperative emesis, electrolyte } \\
\text { imbalance, fever, pain, etc. }\end{array}$ & $7(2.3 \%)$ & Antiemetic, antipyretic, analgesic, diuretic, electrolyte treatments \\
\hline
\end{tabular}

who underwent TUR $P$ as $4.24 \%$ while the readmission rate after laser prostatectomy was $4.27 \%$, which was at a similar rate with the most prevalent readmission cause, hematuria. In the study by Sood et al. (13), the rate of readmission within 30 days after prostatectomy performed with minimally invasive methods was observed as $3.8 \%$.

There are very few studies on hospital readmission after HoLEP and these studies are those which usually include patients having same-day/outpatient surgery, as in who were discharged within the same day after the operation. In the previous HoLEP outpatient surgery series, readmission rates change between $5.5 \%$ and $17.8 \%$ (14-17). In our study, readmission rate after HoLEP was observed as $8.67 \%$ with nonspecific and specific causes. However, if the nonspecific admittance causes were to be excluded, the rate of readmission due to specific causes was observed to be $6.34 \%$, which was in compliance with the literature. Lwin et al. (18) observed the readmission rate as $2.5 \%$ among the patients who had same-day HoLEP performed while the urinary tract infection within 30 days was reported as $4.8 \%$. In another study analyzing the same-day HoLEP effectivity, Abdul-Muhsin et al. (14) reported that $17.8 \%$ of the discharged patients were readmitted with the most prevalent cause of hematuria. This elevation in this rate may be explained with the discharge on the same day as the surgery and thus hematuria follow-up not being performed as necessary. In our study, hematuria, which was a cause of readmission, was observed as $2.3 \%$. If clot retentions were to be included in the hematuria group, hematuria could be accepted as the most prevalent readmission cause in our study. In the same study, Abdul-Muhsin et al. (14) observed that the urinary tract infection history among the patients who were readmitted to the hospital was more prevalent compared to the rest of the group $(p=0.0304)$. In our study, urinary tract infection was observed at the rate of $1.33 \%$ and as the third most prevalent cause among specific causes. In the study carried out by Lee et al. (17), readmission rate after HoLEP was shown as $5.5 \%$. Readmission causes were equally observed as hematuria $(n=1)$, urinary tract infection $(n=1)$, deep vein thrombosis $(n=1)$, and inguinal pain of unknown origin $(n=1)$. In the multivariable analysis, being in the morning operation list [odds ratio (OR): 6.124, 95\% confidence interval $(\mathrm{Cl})$ : 2.526-14.845, $\mathrm{p}<0.001]$ and low enucleated weight of $\leq 40 \mathrm{~g}$ (OR: $3.097,95 \% \mathrm{Cl}: 1.619-5.924, \mathrm{p}=0.001$ ) were determined as the predictive factors for readmission to the hospital (17). When the studies are analyzed, it can be observed that readmission rates and causes after TUR $P$ and HoLEP show similarities.

In our study, multivariate analysis was not performed to determine predictive factors for re-admissions after HoLEP surgery. This can be considered as a limiting factor for our article.

\section{Conclusion}

Most prevalent causes for post-HoLEP readmission are (besides nonspecific causes) hematuria, urinary retention, and urinary tract infection. The rates of these causes were acceptable and were comparable to TUR P. It is important to know the reasons and rates of readmission after HoLEP, to predict early complications after surgery, and to manage these complications.

\section{Ethics}

Ethics Committee Approval: The study protocol was approved by Gazi University Ethical Committee, Turkey (approval number: 2020-150) and complied with the Helsinki Declaration.

Informed Consent: Retrospective study.

Peer-review: Externally and internally peer-reviewed. 


\section{Authorship Contributions}

Surgical and Medical Practices: E.K., S.Y., S.B., L.T., Concept: E.G., S.Ya., L.T., Design: E.G., S.Ya., S.Y., Data Collection or Processing: E.G., E.K., M.Y., Analysis or Interpretation: E.K., S.B., L.T., Literature Search: S.Ya., M.Y., Writing: E.G., M.Y.

Conflict of Interest: No conflict of interest was declared by the authors.

Financial Disclosure: The authors declared that this study received no financial support.

\section{References}

1. Gratzke C, Bachmann A, Descazeaud A, et al. EAU Guidelines on the Assessment of Non-neurogenic Male Lower Urinary Tract Symptoms including Benign Prostatic Obstruction. Eur Urol. 2015;67:1099-1109.

2. Nair SM, Pimentel MA, Gilling PJ. A Review of Laser Treatment for Symptomatic BPH (Benign Prostatic Hyperplasia). Curr Urol Rep. 2016;17:45.

3. Rieken M, Ebinger Mundorff N, Bonkat G, Wyler S, Bachmann A. Complications of laser prostatectomy: a review of recent data. World J Urol. 2010;28:53-62.

4. Xiao KW, Zhou L, He Q, et al. Enucleation of the prostate for benign prostatic hyperplasia thulium laser versus holmium laser: a systematic review and meta-analysis. Lasers Med Sci. 2019;34:815-826.

5. Vincent MW, Gilling PJ. HoLEP has come of age. World J Urol. 2015;33:487-493.

6. Palmisano F, Boeri L, Fontana $M$, et al. Incidence and predictors of readmission within 30 days of transurethral resection of the prostate: a single center European experience. Sci Rep. 2018;8:6575.

7. Schmid M, Chiang HA, Sood A, et al. Causes of hospital readmissions after urologic cancer surgery. Urol Oncol. 2016;34:236.

8. Yalçın S, Yılmaz S, Gazel E, et al. Holmium laser enucleation of the prostate for the treatment of size-independent $\mathrm{BPH}$ :
A single-center experience of 600 cases. Turk J Urol. 2020;46:219-225.

9. Rambachan A, Matulewicz RS, Pilecki M, Kim JYS, Kundu SD. Predictors of readmission following outpatient urological surgery. J Urol. 2014;192:183-188.

10. Gore JL, Lai J, Gilbert SM; Urologic Diseases in America Project. Readmissions in the postoperative period following urinary diversion. World J Urol. 2011;29:79-84.

11. Moschini M, Gandaglia G, Dell'Oglio P, et al. Incidence and Predictors of 30-Day Readmission in Patients Treated With Radical Cystectomy: A Single Center European Experience. Clin Genitourin Cancer. 2016;14:341-346.

12. Moschini M, Gandaglia G, Fossati N, et al. Incidence and Predictors of 30-Day Readmission After RobotAssisted Radical Prostatectomy. Clin Genitourin Cancer. 2017;15:67-71.

13. Sood A, Meyer CP, Abdollah F, et al. Minimally invasive surgery and its impact on 30-day postoperative complications, unplanned readmissions and mortality. $\mathrm{Br} \mathrm{J}$ Surg. 2017; 104:1372-1381.

14. Abdul-Muhsin $\mathrm{H}$, Critchlow $\mathrm{W}$, Navaratnam $\mathrm{A}$, et al. Feasibility of holmium laser enucleation of the prostate as a 1-day surgery. World J Urol. 2020;38:1017-1025.

15. Comat V, Marquette T, Sutter W, et al Day-Case Holmium Laser Enucleation of the Prostate: Prospective Evaluation of 90 Consecutive Cases. J Endourol. 2017;31:1056-1061.

16. Larner TRG, Agarwal D, Costello AJ. Day-case holmium laser enucleation of the prostate for gland volumes of $<60$ $\mathrm{mL}$ : early experience. BJU Int. 2003;91:61-64.

17. Lee SM, Gordon K, McMillan R, Crystal F, Acher P. Daycase holmium laser enucleation of the prostate: feasibility, safety and predictive factors. Ann R Coll Surg Engl. 2018; $100: 475-479$.

18. Lwin AA, Zeng J, Evans $P$, et al. Holmium Laser Enucleation of the Prostate is Safe and Feasible as a Same Day Surgery. Urology. 2020;138:119-124. 